\title{
ACTIVATION OF MATERIALS PROPOSED FOR USE IN SUPERCONDUCTING LINAC APPLICATIONS
}

\author{
A.L. Hanson, C.L. Snead, Jr., G.A. Greene \\ Brookhaven National Laboratory \\ K.C. Dominic Chan \\ Los Alamos National Laboratory \\ H. Safa \\ C.E. Saclay, France
}

JANUARY 1998

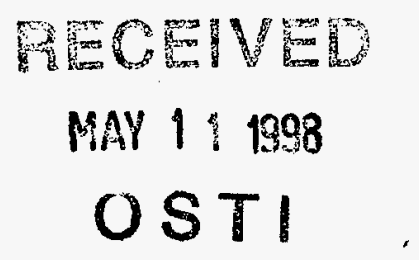

Brookhaven National Laboratory

Brookhaven Science Associates

Upton, New York 11973 


\title{
ACTIVATION OF MATERIALS PROPOSED FOR USE IN SUPERCONDUCTING LINAC APPLICATIONS
}

\author{
A.L. Hanson, C.L. Snead, Jr., G.A. Greene \\ Brookhaven National Laboratory \\ K.C. Dominic Chan \\ Los Alamos National Laboratory \\ H. Safa \\ C.E. Saclay, France
}

JANUARY 1998

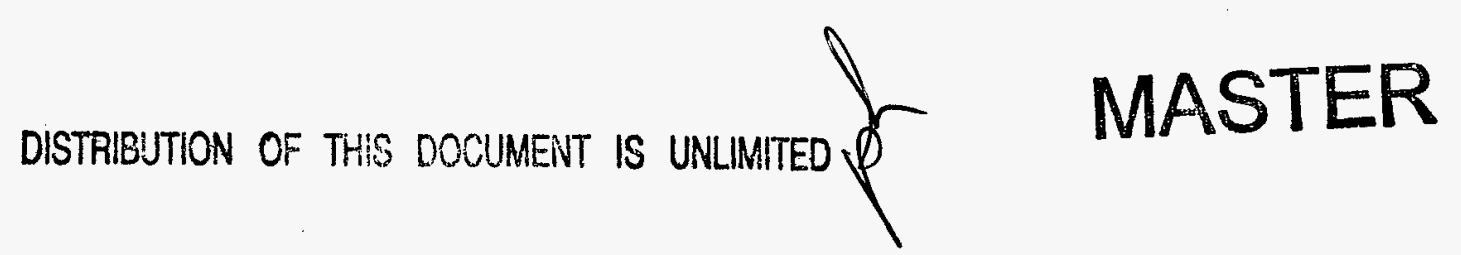

Department of Advanced Technology

Brookhaven National Laboratory

Brookhaven Science Associates

Upton, New York 11973

Under Contract No. DE-AC02-98CH10886 with the

UNITED STATES DEPARTMENT OF ENERGY 


\section{DISCLAIMER}

This report was prepared as an account of work sponsored by an agency of the United States Government. Neither the United States Government nor any agency thereof, nor any of their employees, makes any warranty, express or implied, or assumes any legal liability or responsibility for the accuracy, completeness, or usefulness of any information, apparatus, product, or process disclosed, or represents that its use would not infringe privately owned rights. Reference herein to any specific commercial product, process, or service by trade name, trademark, manufacturer, or otherwise does not necessarily constitute or imply its endorsement, recommendation, or favoring by the United States Government or any agency thereof. The views and opinions of authors expressed herein do not necessarily state or reflect those of the United States Government or any agency thereof. 
Activation of Materials Proposed for Use in Superconducting Lina Applications

A.L. Hanson, C.L. Snead, Jr., G.A. Greene Brookhaven National Laboratory

K.C. Dominic Chan

Los Alamos National Laboratory

H. Sofa

C.E. Saclay, France

ABSTRACT

Samples of construction materials proposed for use in both superconducting and conventional high-power linear accelerators have been activated with 800 and $2000 \mathrm{MeV}$ protons to study the decay characteristics of these activated materials. Irradiation times ranged from 10 minutes to 18.67 hours. The decay characteristics of these activated materials were measured and compared to calculated decay curves based on simplified assumptions.

1 


\section{INTRODUCTION}

Radiological safety can impact the availability of accelerators because of activation of the structural materials from stray beam during normal operations or from beam mis-steering accidents. The concerns of radiological safety are magnified for the high-power accelerators that are being designed for the accelerator production of tritium (APT in the U.S.A. or TRISPAL in France), for the accelerator transmutation of waste, and for neutron spallation sources. In particular, the present APT accelerator is designed to deliver $100 \mathrm{~mA}$ of $1.7-\mathrm{GeV}$ protons on target, $170 \mathrm{MW}$ of power. These currents are several orders of magnitude higher than the currents of conventional research accelerators.

The normal operation of any accelerator has some level of stray beam (beam halo) associated with it. This stray beam strikes the accelerator materials and can result in activation of those materials. For the APT design, this value is on the order of $0.2 \mathrm{nA} / \mathrm{m}$. Additionally, there is always a chance for a beam mis-steering accident where a high-current particle beam strikes the wall of the accelerator and activates it. The level of the radiation field and its decay from activated materials will determine how soon after shutdown the accelerator can be serviced. Each of the two types of activation results in different activation and decay characteristics. The activation from beam halo is a slow, continual process over the time of accelerator operation. In contrast, the beam dump is a fast, short (probably on the order of milliseconds with these beam powers) term process. At shutdown the initial radiation level from the beam mis-steering activation would probably be higher than the level from the beam halo activation. However, it will probably decay faster than the activation from the beam halo. The longer decay from the halo activation would be due to longer-lived isotopes being created in sufficient quantities. This assumption will need to be confirmed.

The radiation level from proton activation could pose significant problems to repair and scheduled maintenance if the actual radiation levels are substantially higher and/or the decay is slower than predicted from the calculations. Because there is no comprehensive set of measured cross-section data for the activation of materials to compare with calculated 
cross sections (and hence activation), samples of several materials expected to be present in high power accelerators were activated with 800 and $2000 \mathrm{MeV}$ protons and the measured decay curves compared to decay curves based on calculated production cross sections from a series of initial scoping calculations.

\section{MEASUREMENTS}

Samples of materials being considered for both room temperature and superconducting accelerator systems were prepared and irradiated with 800 and $2000 \mathrm{MeV}$ protons. Two sets of samples were prepared. The first set consisted of niobium cut into samples $1 \mathrm{~cm} \mathrm{x}$ $1 \mathrm{~cm} \times 0.2 \mathrm{~cm}(1.74 \mathrm{~g})$. The second set consisted of niobium, copper, carbon steel, stainless steel, and aluminum, cut into TEM (transmission electron microscopy) standard sized disks, $3 \mathrm{~mm}$ dia. $\times 0.254 \mathrm{~mm}$ thick. The masses of these samples were $0.015 \mathrm{~g}, 0.016 \mathrm{~g}, 0.014 \mathrm{~g}$, $0.014 \mathrm{~g}$, and $0.0049 \mathrm{~g}$, for the niobium, copper, carbon steel, stainless steel, and aluminum, respectively. Additionally small samples of niobium-titanium superconducting wire (with a copper coating) were included which consisted of pieces $1 \mathrm{~cm}$ long by $0.1 \mathrm{~mm}$ diameter; the weight being $0.032 \mathrm{~g}$.

The proton irradiations were performed at the Laboratorie National Saturne located at CEA/Saclay, France. The samples were mounted between two aluminum dosimetry foils along with three niobium samples that were cut for RRR (residual resistivity ratio) measurements. The entire package was then mounted in the facility's "Doigt de Gant". This Doigt de Gant is a pneumatically driven sample holder that holds 8 -cm-diameter disks. It is located at the extraction 2 of the accelerator before any beam line optics, figure 1. The shape of the beam at the specimen position was elliptical with the long axis being vertical. The beam spot is $3.5 \times 1.5 \mathrm{~cm}^{2}$ resulting in a nonuniform gaussian beam spot of $5.25 \mathrm{~cm}^{2}$. These samples were mounted in sets of 10 , two rows of five mounted in the vertical direction.

The irradiations of the niobium pieces and the TEM disks were carried out parasitically during another experiment, the APT/TRISPAL sponsored $n / p$ experiment. This $n / p$ ex- 
periment required short irradiations (one hour of irradiation followed by seven hours of cool down) over a period of three weeks. These samples were inserted in the beam when the beam was not being used for the $n / p$ experiment.

After irradiation, the samples were removed from the Doigt de Gant and from the aluminum dosimetry foils. The decay curves for the samples were measured with an ionization chamber while the aluminum dosimetry foils were counted for the ${ }^{24} \mathrm{Na}$ activity with an intrinsic germanium detector. The ${ }^{24} \mathrm{Na}$ activity is used to determine the total number of protons that struck the targets.

The 1.7-g niobium samples were irradiated at either $800 \mathrm{MeV}$ for 33 minutes to a fluence of $2.8 \times 10^{13} \mathrm{p} / \mathrm{cm}^{2}$ or at $2000 \mathrm{MeV}$ for 10 minutes to a fluence of $6.4 x 10^{12} \mathrm{p} / \mathrm{cm}^{2}$. The TEM disks irradiated with $800 \mathrm{MeV}$ and $2000 \mathrm{MeV}$ protons. The $800-\mathrm{MeV}$ irradiations were for 18.67 hours, and the samples received a fluence of $1.7 \times 10^{15} \mathrm{p} / \mathrm{cm}^{2}$. The samples irradiated with the $2000-\mathrm{MeV}$ protons received a fluence of $0.45 \times 10^{15} \mathrm{p} / \mathrm{cm}^{2}$ in 3.5 hours.

\section{CALCULATIONS}

The measured decay curves will be compared to calculated decay curves. In order to obtain a decay curve, the probability for producing each isotope created during the irradiation is calculated with Monte Carlo high-energy physics transport computer code, LAHET [Pr89]. The probability for producing each isotope, coupled with the length of irradiation and the half lives of each isotope, can be used to calculate the total activity and decay curves for each sample. In order to calculate the dose-rate and the dose rate decay curve, the energy and number of the gamma rays must be folded into the calculation along with the appropriate dose conversion factors. Since the gamma ray spectra can change significantly with time (due to changes in the mix of radioisotopes), the dose-rate decay curve may not necessarily track with the activity decay curve. For the present version of this report, we will necessarily rely only on the calculated activity of the isotopes. Work in FY98 includes incorporating the output of the LAHET coded with the ORIGEN-2M [Cr83,Co95] and CINDER90 [Wi93] 
codes both of which explicitly account for time-dependent build-up and decay of the isotopes produced. When completed this will result in a computation that can be used to predict the dose-rate decay curve.

At present the LAHET calculations used for this report should be considered to be very preliminary in that the experimental configurations were not explicitly modelled; i.e. the calculations assumed a bare cube of material $1 \mathrm{~cm} x 1 \mathrm{~cm} x 1 \mathrm{~cm}$ with no surroundings. They were carried out for only $800-\mathrm{MeV}$ protons incident on the niobium, copper, iron, and aluminum and 2000-MeV protons incident on niobium. Most of the calculations were only 50,000 proton histories for each target. One calculation was made for $800-\mathrm{MeV}$ protons on niobium which used $1,000,000$ histories. The output used from these calculations were the production of a particular isotope per proton: $\left(\frac{P R O D}{P}\right)$. The minimum values of $\left(\frac{P R O D}{P}\right)$ that the code can calculate depends on the number of histories, $N:\left(\frac{P R O D}{P}\right)_{\min }=\frac{1}{N}$. This implies that the accuracy of $\left(\frac{P R O D}{P}\right)$ for the calculation of any particular isotope improves as the number of histories is increased. Also, as $\mathrm{N}$ increases, more isotopes are calculated to be produced. However, the isotopes that were missed by considering only 50,000 histories will only make a small contribution to the overall activity.

Calculating the activity in a sample irradiated with high-energy protons is a moderately complicated process. First there is a large number of isotopes generated. Secondly, the activity at any time during or after the irradiation depends on: the time and characteristics of the irradiation; the half-life of each isotope; the production cross section; the decay time; and the daughter products. Decay chains can be used to determine which metastable states are populated during the decay, but one must make some assumptions as to which isotopes are produced in their metastable states during the spallation process. For the present version of this report, it will be assumed that during the spallation process, isotopes will be created in their metastable states.

The large number of isotopes generated is demonstrated in Figures 2 and 3 . These figures show the distribution of isotopes, as calculated with the LAHET code, created by 
the irradiation of niobium with 800 and $2000 \mathrm{MeV}$ protons. The total number of isotopes created when niobium is irradiated with $800-\mathrm{MeV}$ protons is greater than 140 and when the niobium irradiated with $2000-\mathrm{MeV}$ protons the number is greater than 210 .

The activity $\left(\mathrm{A}_{I}(0)\right)$ for the direct generation of any specific isotope $I$, at the end of an irradiation of $\mathbf{t}$ seconds is calculated with:

$$
A_{I}(0)=P *\left(\frac{P R O D}{P}\right)_{I} *\left(\frac{e^{\left(-\lambda_{I} * t\right)}}{t}\right)
$$

where:

$\mathrm{P}=$ number of protons

$\left(\frac{P R O D}{P}\right)_{I}=$ the production of the isotope of interest I per incident proton (calculated by LAHET)

$I=$ decay constant for isotope $I$ (per second)

Equation (1) assumes that the proton beam current is relatively steady over the time of the irradiation. This equation has two limits, one when the half-life of a particular isotope is short with respect to the irradiation time $\left(t_{1 / 2, I} \ll t\right)$ and the other when the half-life is long with respect to the irradiation time $\left(t_{1 / 2, I} \gg t\right)$. For the first case $A_{I}(0)$ can be approximated with:

$$
A_{I}(0)=\frac{P *\left(\frac{P R O D}{P}\right)_{I}}{t}
$$

and for the second case

$$
A_{I}(0)=P *\left(\frac{P R O D}{P}\right)_{I} * \lambda_{I}
$$

In the first case, the activity is independent of the half life because the creation of the particular isotope has reached "saturation" so the creation of the isotope equals the loss due to decay. In the second case the activity is independent of the irradiation time because the decay rate is insignificant over the time of the irradiation. This means that the actual 
production depends only on how many protons strike the target and is totally independent of the irradiation time, $t$.

The activity of the isotope $\mathrm{A}_{I}$ after decaying DT seconds is:

$$
A_{I}(D T)=A_{I}(0) * e^{\left(-\lambda_{I} * D T\right)}
$$

Many of the spallation products for the elements of interest here have daughter isotopes, so the calculation of the activity must include them. The activities for the daughter products, up to three daughters, are given below. First, however, we will define a few terms:

$$
\begin{aligned}
& C 1=\frac{\lambda}{\lambda_{1} *\left(\lambda_{1}-\lambda\right)} \\
& C 2=\frac{1}{\left(\lambda_{1}-\lambda\right) *\left(\lambda_{2}-\lambda\right)}-\frac{1}{\lambda_{1} * \lambda_{2}}-\frac{C 1}{\left(\lambda_{2}-\lambda_{1}\right)}, \\
& C 3=\frac{1}{\left(\lambda_{1}-\lambda\right) *\left(\lambda_{2}-\lambda\right) *\left(\lambda_{3}-\lambda\right)}-\frac{1}{\lambda_{1} * \lambda_{2} * \lambda_{3}}-\frac{C 1}{\left(\lambda_{2}-\lambda_{1}\right) *\left(\lambda_{3}-\lambda_{1}\right)}-\frac{C 2}{\left(\lambda_{3}-\lambda_{2}\right)}, \\
& B 1=\frac{\lambda_{1} * A(0)}{\left(\lambda_{1}-\lambda\right) *\left(\lambda_{2}-\lambda\right)} \\
& B 2=\frac{A_{1}(0)}{\left(\lambda_{2}-\lambda_{1}\right)}-\frac{\lambda_{1} * A(0)}{\left(\lambda_{1}-\lambda\right) *\left(\lambda_{2}-\lambda_{1}\right)}, \\
& B 3=\frac{\lambda_{1} * A(0)}{\left(\lambda_{2}-\lambda\right) *\left(\lambda_{2}-\lambda_{1}\right)}-\frac{A_{1}(0)}{\left(\lambda_{2}-\lambda_{1}\right)}+\frac{A_{2}(0)}{\lambda_{2}}
\end{aligned}
$$

and

$$
B 4=\frac{A_{3}(0)}{\lambda_{3}}-\lambda_{2} *\left[\frac{B 1}{\left(\lambda_{3}-\lambda\right)}+\frac{B 2}{\left(\lambda_{3}-\lambda_{1}\right)}+\frac{B 3}{\left(\lambda_{3}-\lambda_{2}\right)}\right] .
$$

From here on, it is assumed that each daughter product is the daughter of isotope $\mathrm{I}$, so the subscript $I$ is dropped. The first daughter is denoted with the subscript " 1 ", the second 
with "2" and the third with "3". The activity of the first daughter, at the end of irradiation is:

$$
A_{1}(0)=\frac{\lambda_{1} * P}{T} *\left[\frac{1}{\lambda_{1}}-\frac{e^{(-\lambda * T)}}{\left(\lambda_{1}-\lambda\right)}+C 1 * e^{\left(-\lambda_{1} * T\right)}\right]
$$

and after DT seconds of decay is given by

$$
A_{1}(D T)=\frac{A(0) * \lambda_{1}}{\left(\lambda_{1}-\lambda\right)} *\left(e^{(-\lambda * D T)}-e^{\left(-\lambda_{1} * D T\right)}\right)+A_{1}(0) * e^{\left(-\lambda_{1} * D T\right)} .
$$

The activity of the second daughter at the end of the irradiation is:

$$
A_{2}(0)=\frac{P * \lambda_{1} * \lambda_{2}}{T} *\left[\frac{1}{\lambda_{1} * \lambda_{2}}-\frac{e^{(-\lambda * T)}}{\left(\lambda_{1}-\lambda\right) *\left(\lambda_{2}-\lambda\right)}+\frac{C 1 * e^{\left(-\lambda_{1} * T\right)}}{\left(\lambda_{2}-\lambda_{1}\right)}+C 2 * e^{\left(-\lambda_{2} * T\right)}\right] .
$$

After DT seconds of decay this activity is:

$$
\begin{gathered}
A_{2}(D T)=\lambda_{1} \lambda_{2} *\left[\frac{B 3 * e^{\left(-\lambda_{2} * D T\right)}}{\lambda_{1}}+\frac{A(0) * e^{(-\lambda * D T)}}{\left(\lambda_{1}-\lambda\right) *\left(\lambda_{2}-\lambda\right)}-\frac{A(0) * e^{\left(-\lambda_{1} * D T\right)}}{\left(\lambda_{1}-\lambda\right) *\left(\lambda_{2}-\lambda_{1}\right)}\right. \\
\left.+\frac{A_{1}(0) * e^{\left(-\lambda_{1} * D T\right)}}{\lambda_{1} *\left(\lambda_{2}-\lambda_{1}\right)}\right] .
\end{gathered}
$$

The activity of the third daughter at the end of the irradiation is:

$$
\begin{aligned}
A_{3}(0)= & \frac{P * \lambda_{1} * \lambda_{2} * \lambda_{3}}{T} *\left[\frac{1}{\lambda_{1} * \lambda_{2} * \lambda_{3}}-\frac{e^{(-\lambda * T)}}{\left(\lambda_{1}-\lambda\right) *\left(\lambda_{2}-\lambda\right) *\left(\lambda_{3}-\lambda\right)}\right. \\
& \left.+\frac{C 1 * e^{\left(-\lambda_{1} * T\right)}}{\left(\lambda_{2}-\lambda_{1}\right) *\left(\lambda_{3}-\lambda_{1}\right)}+\frac{C 2 * e^{\left(-\lambda_{2} * T\right)}}{\left(\lambda_{3}-\lambda_{2}\right)}+C 3 * e^{\left(-\lambda_{3} * T\right)}\right] .
\end{aligned}
$$

The activity of the third daughter after DT seconds is:

$$
A_{3}(D T)=\lambda_{2} * \lambda_{3} *\left[\frac{B 1 * e^{(-\lambda * D T)}}{\left(\lambda_{3}-\lambda\right)}+\frac{B 2 * e^{\left(-\lambda_{1} * D T\right)}}{\left(\lambda_{3}-\lambda_{1}\right)}+\frac{B 3 * e^{\left(-\lambda_{2} * D T\right)}}{\left(\lambda_{3}-\lambda_{2}\right)}+\frac{B 4 * e^{\left(-\lambda_{3} * D T\right)}}{\lambda_{2}}\right] .
$$




\section{DECAY CURVES}

Decay curves for the dose rates from each TEM sample irradiated with $800 \mathrm{MeV}$ protons are shown in figure 4 . There are several interesting features of the curves in this figure. First, the aluminum decays faster than any other element, the only significant calculated products being ${ }^{24} \mathrm{Na}$ and ${ }^{22} \mathrm{Na}$. Second, there was no significant difference between the iron and stainless steel. This implies that the alloying elements have insignificant contributions to changes in the activity. Third, the copper activates to a larger dose rate than the iron and the niobium activates to a greater extent than either. However, even though the niobium starts with a higher value than the copper, it decays more rapidly than the copper. Third, the niobium-titanium wire starts with an activity close to the niobium, but has a decay curve closer to the copper. This is because this wire is clad in copper.

One test of the calculational models is to compare calculated decay curves with measured decay curves recognizing the caveats noted earlier. The decay curves of the activity depend on several factors such as the energy of the bombarding protons and the length and history of the irradiation. The proton energy determines the cross section for the production of each isotopes and the time history of the irradiation determine which isotopes will saturate during the bombardment, the saturation depending on whether the half-life of the isotope is short compared to the irradiation time. The dose rate is complicated by an energy dependence. The spectra of the isotopes need to be folded into the calculation. At the present time this has not been done, and it is assumed that the decay curve of the dose rate can be approximated with the decay curve of the activity. The energy dependence will be included in the planned calculations utilizing the LAHET plus ORIGEN-2M and CINDER90 codes for a detailed simulation of the experiments.

If we only consider the activity in the sample, it is interesting to define saturation as $90 \%$ of unity. For the activation of niobium with $2000-\mathrm{MeV}$ protons, $15 \%$ of the isotopes generated will be saturated when niobium is irradiated for 10 minutes, but $43 \%$ will be saturated after a $3.5 \mathrm{~h}$ irradiation. These numbers were based on the present LAHET calculations. Because 
the activity (along with the saturation) depends on the half-life of the isotopes, the fraction of activity resulting from saturated isotopes will be different from the total number of isotopes having become saturated. For the $2000-\mathrm{MeV}$ proton irradiation of niobium for 10 minutes and 3.5 hours, $68 \%$ and $78 \%$ of the activity (when the beam is turned off) is from saturated isotopes, respectively. Likewise for the two irradiation times for the $800-\mathrm{MeV}$ irradiations, $33 \mathrm{~min}$ and $18.67 \mathrm{~h}$, the percentage of isotopes having reached saturation is $21 \%$ and $56 \%$, respectively, and by percentage by simple activity is $79 \%$ and $85 \%$, respectively.

The measured and calculated decay curves for the $1.7 \mathrm{~g}$ niobium samples are shown in figures 5 and 6 for activation with $800 \mathrm{MeV}$ and $2000 \mathrm{MeV}$ protons, respectively. In order to compare with the calculated decay curves, both are plotted on relative scales. For the $800-\mathrm{MeV}$ protons, the data acquisition started two minutes after the beam was turned off and for the $2000-\mathrm{MeV}$ protons, the data acquisition was started six minutes after the beam was turned off. In the measured curves 1 is equivalent to $1 \mathrm{mr} / \mathrm{h} / \mathrm{g}$ for both 800 and 2000 $\mathrm{MeV}$ protons.

The measured and calculated decay curves for the activated TEM disks are shown in figures 7 through 11 . Figure 12 shows the relative decay curves for the materials irradiated with $2000-\mathrm{MeV}$ protons. As can be seen from these figures, the agreement between calculated and measured decay curves are very good for the activation of niobium and aluminum. However, the agreement between the calculated and measured decay curves was not very good for the iron and copper. The activation of the iron and copper result is 10 and $20 \%$, respectively, activity from phosphorous and sulfur isotopes. These isotopes produce no gamma rays. The seeming disagreement between the measured and calculated data may be in part due to this.

One purpose of this work is to help understand the potential radiation hazards that may interfere with maintenance and repair of a high-power accelerator following a shutdown. One aspect of this issue is how quickly the activity will decay following the shutdown. Since the decay curve changes with time of irradiation, it is instructive to plot the effective half-life 
as a function of irradiation time. Figure 13 shows this effective half-life for niobium, iron, and copper as a function of irradiation time, for $800-\mathrm{MeV}$ protons (the aluminum decays rapidly). These curves were generated assuming constant uninterrupted proton irradiation during the time. The "humps" in the curves in this figure are due to the saturation of longer lived isotopes as the irradiation time gets longer.

\section{CONCLUSIONS}

The prediction of decay curves of materials irradiated with $\mathrm{GeV}$ protons is not necessarily a straightforward issue because of the large number of isotopes that are created along with the gamma-ray spectra. The uncertainties in the calculation of key isotopes could have significant impacts on expected out-of-service time if the calculated decay curves significantly underpredict the level of radiation around components requiring service. This issue is enhanced for the extremely high-power accelerators such as the one being designed for the APT.

In comparing the calculated activity decay curves to the measured dose rate curves, the curves for the activation of aluminum and niobium agree well. However, the decay curves for copper and iron evidenced poor agreement. In the case of the activation of aluminum, the decay is dominated by one isotope, ${ }^{24} \mathrm{Na}$. In the case of niobium, most of the isotopes emit gamma rays in the $\mathrm{MeV}$ energy range. For both copper and iron, there is a significant creation of phosphorus and sulfur isotopes with either no or low-energy gamma rays. Therefore, until a more complete analysis is performed, including an LCS simulation of the actual experiments followed by analyses with the ORIGEN-2M and CINDER90 isotope build-up and decay codes, this seeming mismatch may be an artifact of the present comparison methodology. 


\section{REFERENCES}

Pr89 R.E. Prael and H. Lichtenstein, User Guide to LCS: the LAHET Code System, LANL Report LA-UR 89-3014 (1989).

Co95 D. Cokinos and M. Todosow, "Validation of the Methods Used in the Nuclear Design of the APT SILC Target/Blanket System", BNL, APT/077315/O322.

Cr83 A.G. Croff, "Origen1: A Versatile Computer Code for Calculating the Nuclide Compositions and Characteristics of Nuclear Materials", Nuclear Technology 62 (1983) 335-352.

Wi93 W.B. Wilson, et al., "Accelerator Transmutation Studies at Los Alamos with LAHET, MCNP and CINDER90", LANL, LA-UR-93-3080, 1993. 


\section{FIGURES}

Figure 1. Layout of the Saturne Accelerator showing the location of the Doigt de Gant at Extraction 2.

Figure 2. Distribution of isotopes created by the irradiation of niobium with $800 \mathrm{MeV}$ protons.

Figure 3. Distribution of isotopes created by the irradiation of niobium with $2000 \mathrm{MeV}$ protons.

Figure 4. Comparison of the measured dose rates for five targets when irradiated with $800 \mathrm{MeV}$ protons for 18.7 hours.

Figure 5. Comparison of the measured decay curve to the calculated decay curve for the 33 minute irradiation of niobium with $800 \mathrm{MeV}$ protons.

Figure 6. Comparison of the measured decay curve to the calculated decay curve for the 10 minute irradiation of niobium with $2000 \mathrm{MeV}$ protons.

Figure 7. Comparison of the measured decay curve to the calculated decay curve for the 18.7 hour irradiation of aluminum with $800 \mathrm{MeV}$ protons.

Figure 8. Comparison of the measured decay curve to the calculated decay curve for the 18.7 hour irradiation of iron with $800 \mathrm{MeV}$ protons.

Figure 9. Comparison of the measured decay curve to the calculated decay curve for the 18.7 hour irradiation of copper with $800 \mathrm{MeV}$ protons.

Figure 10. Comparison of the measured decay curve to the calculated decay curve for the 18.7 hour irradiation of niobium with $800 \mathrm{MeV}$ protons.

Figure 11. Comparison of the measured decay curve to the calculated decay curve for the 13.5 hour irradiation of niobium with $2000 \mathrm{MeV}$ protons.

Figure 12. Comparison of the measured dose rates for five targets when irradiated with $2000 \mathrm{MeV}$ protons for 3.5 hours.

Figure 13. Calculated effective half-life as a function of irradiation time for the irradiation of iron, copper, and niobium with $800 \mathrm{MeV}$ proton. 


\section{SATURNE Accelerator facility}

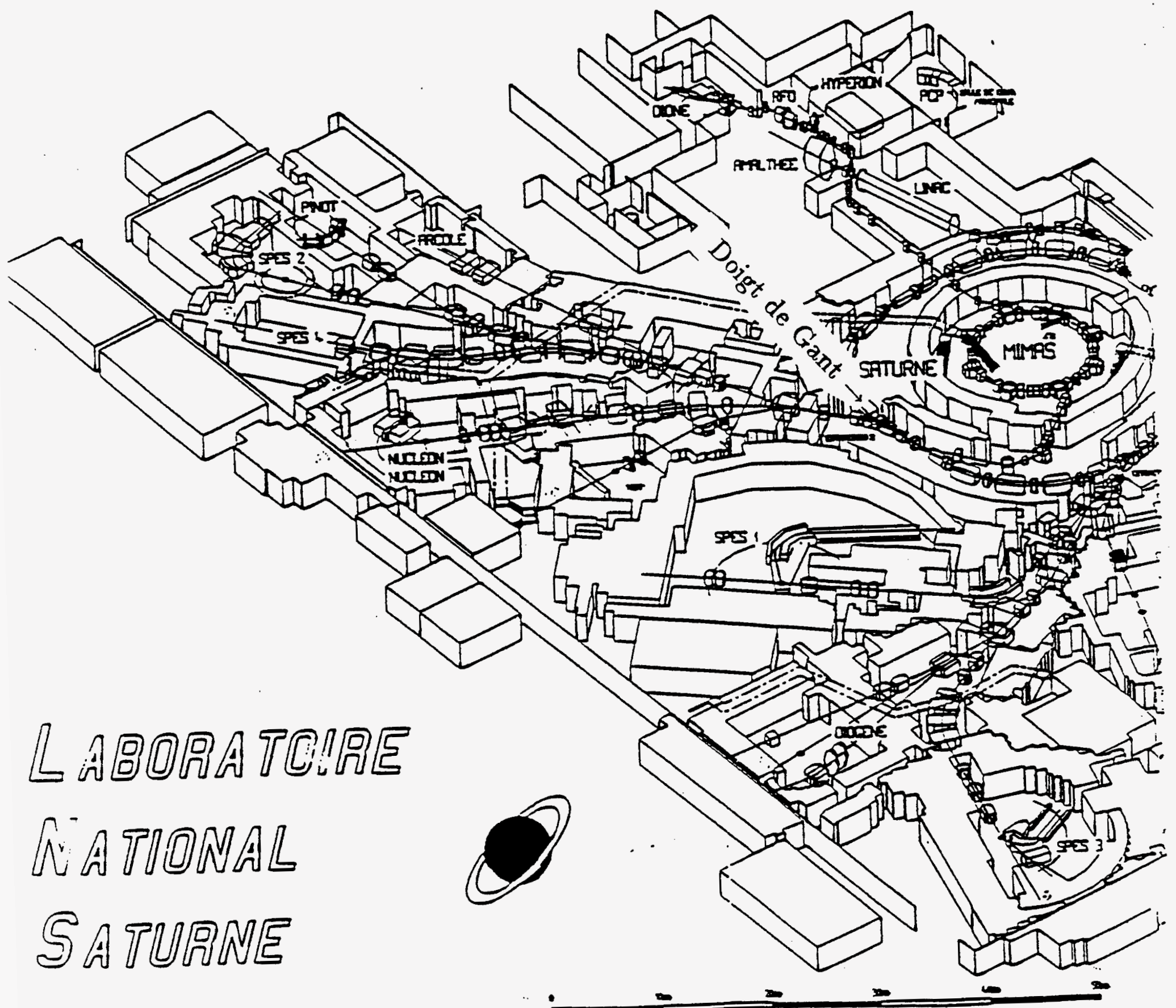

Figure 1 


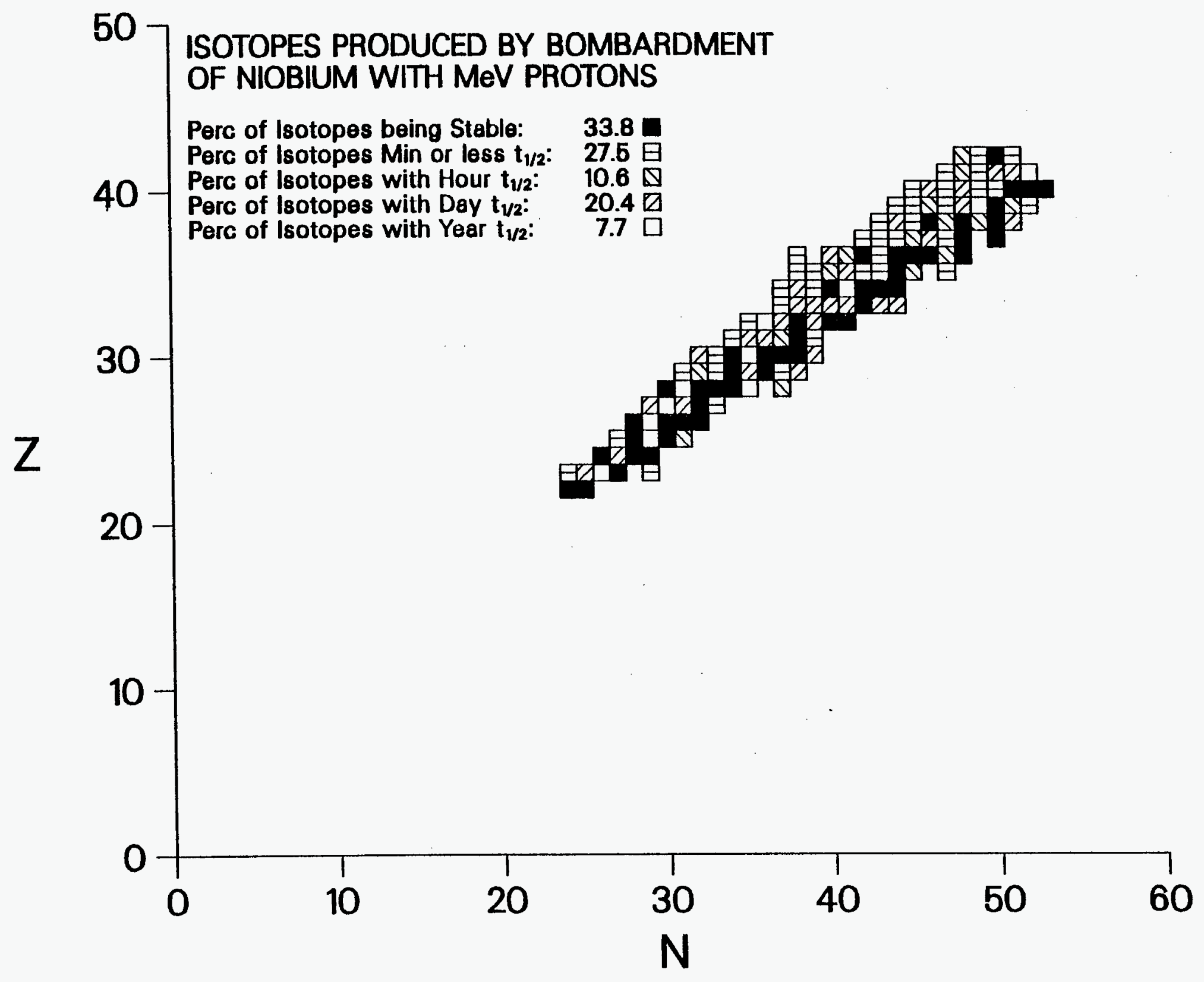

Figure 2 


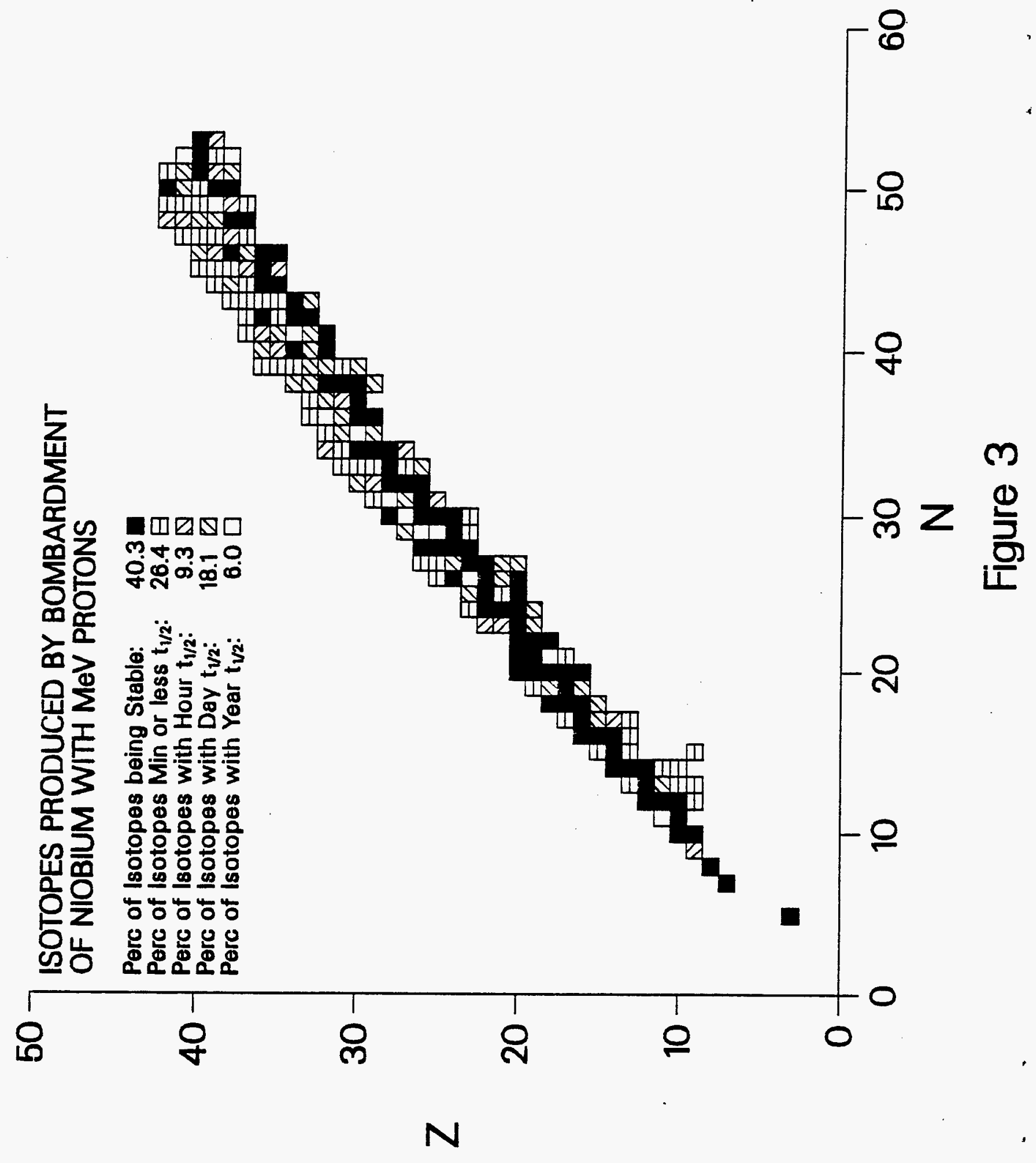


Measured Dose Rate Normalized to Mass $800 \mathrm{MeV}$ Protons; 18.67h Irradiation

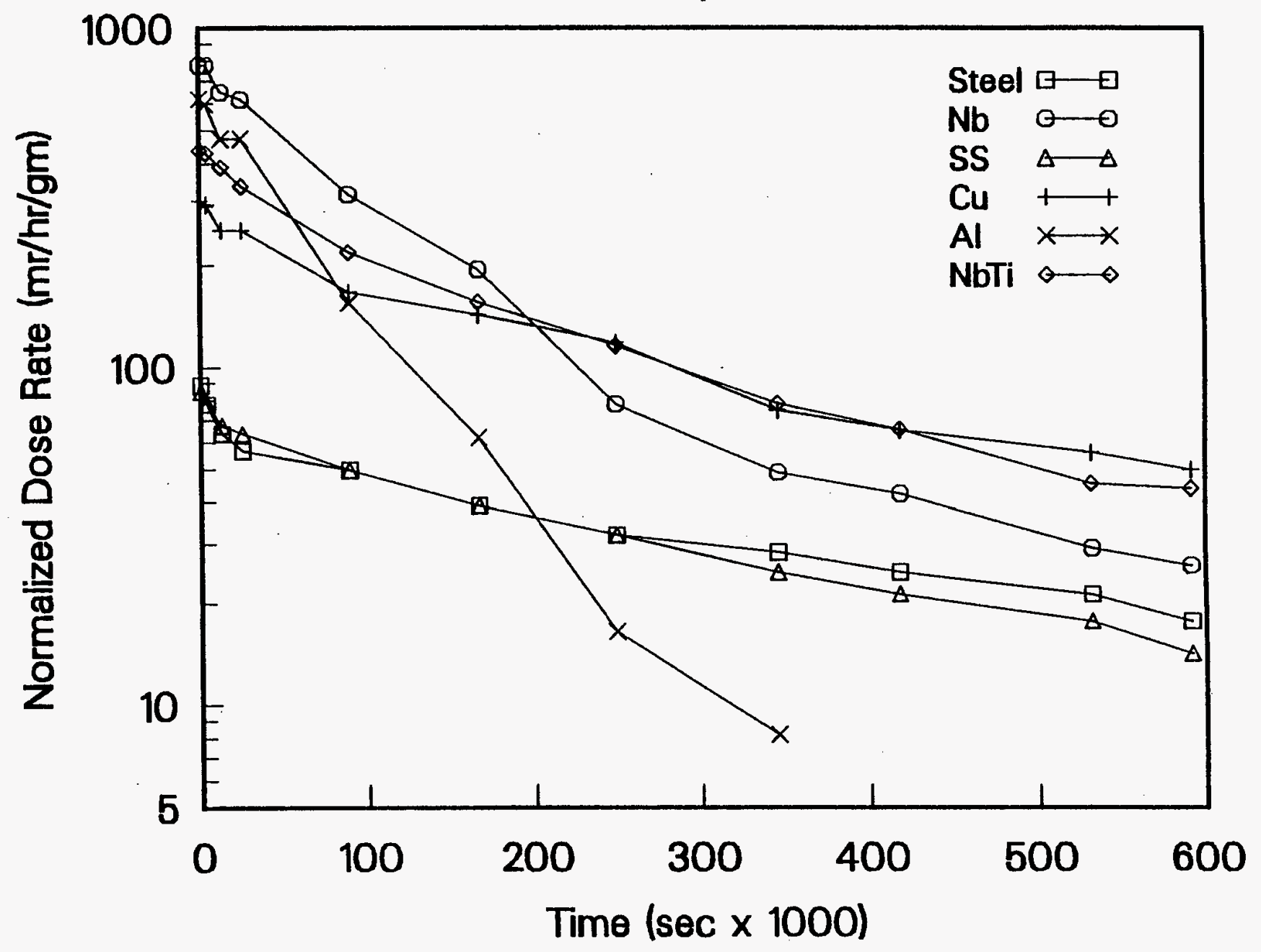

Figure 4 
Measured and Calculated Decay Curves Normalized at 2 minutes $800 \mathrm{MeV}$ Protons on Niobium; $33 \mathrm{~min}$ Irrad; $2.5 \mathrm{E} 13 \mathrm{p} / \mathrm{cm} 2$

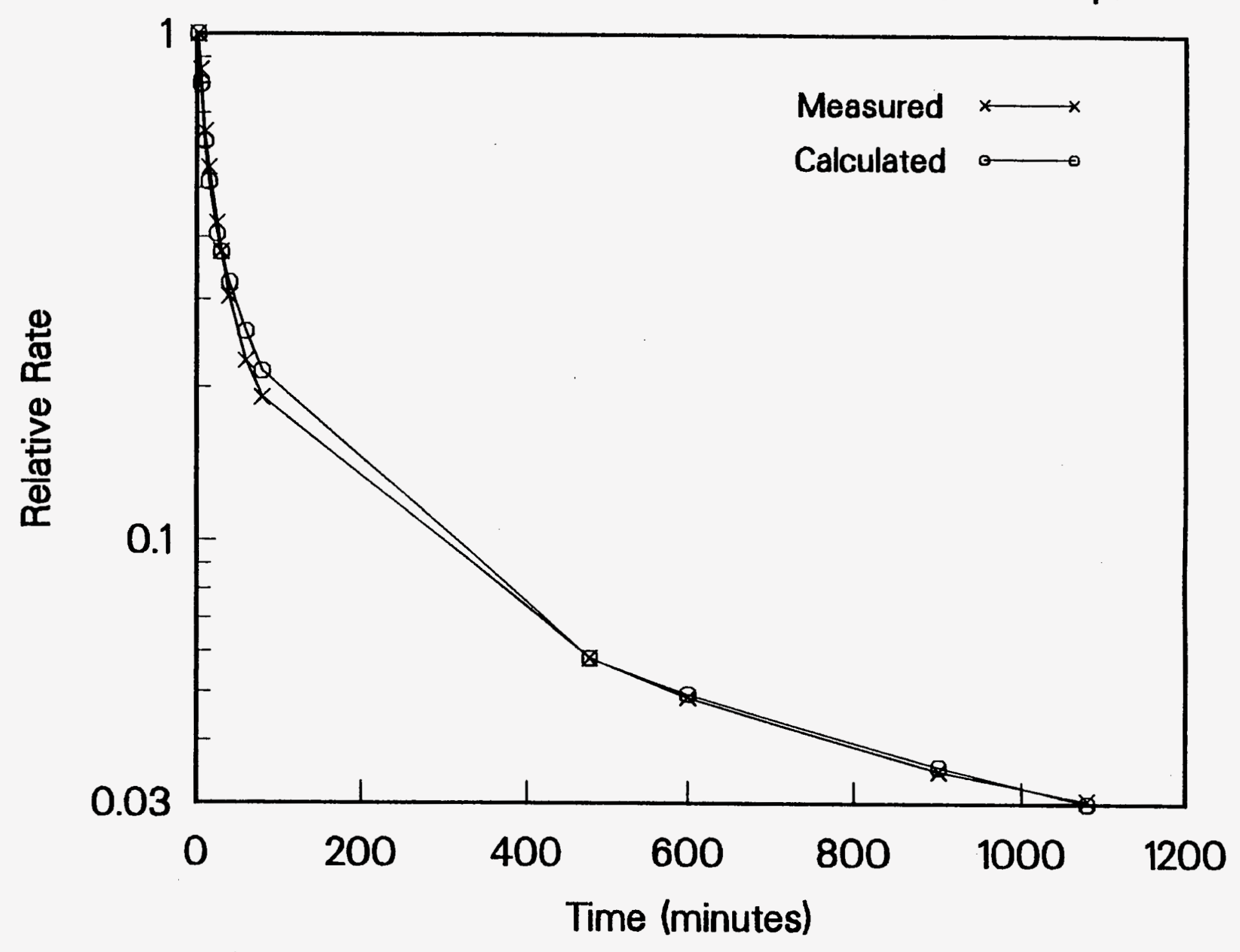

Figure 5 
Measured and Calculated Decay Curves Normalized at 6 minutes $2000 \mathrm{MeV}$ Protons on Niobium; $10 \mathrm{~min}$ Irrad; $1 . E 13$ p/cm2

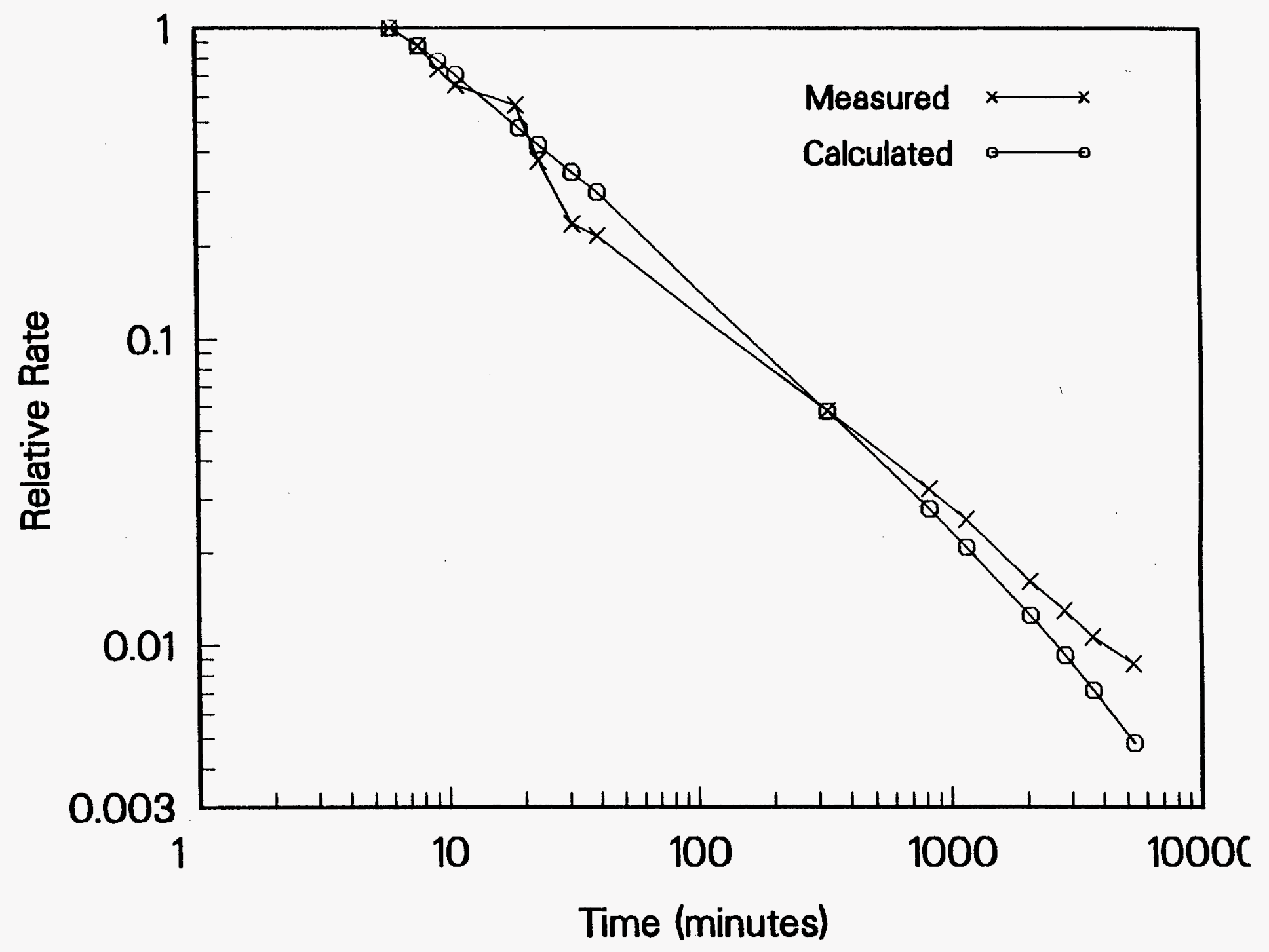

Figure 6 


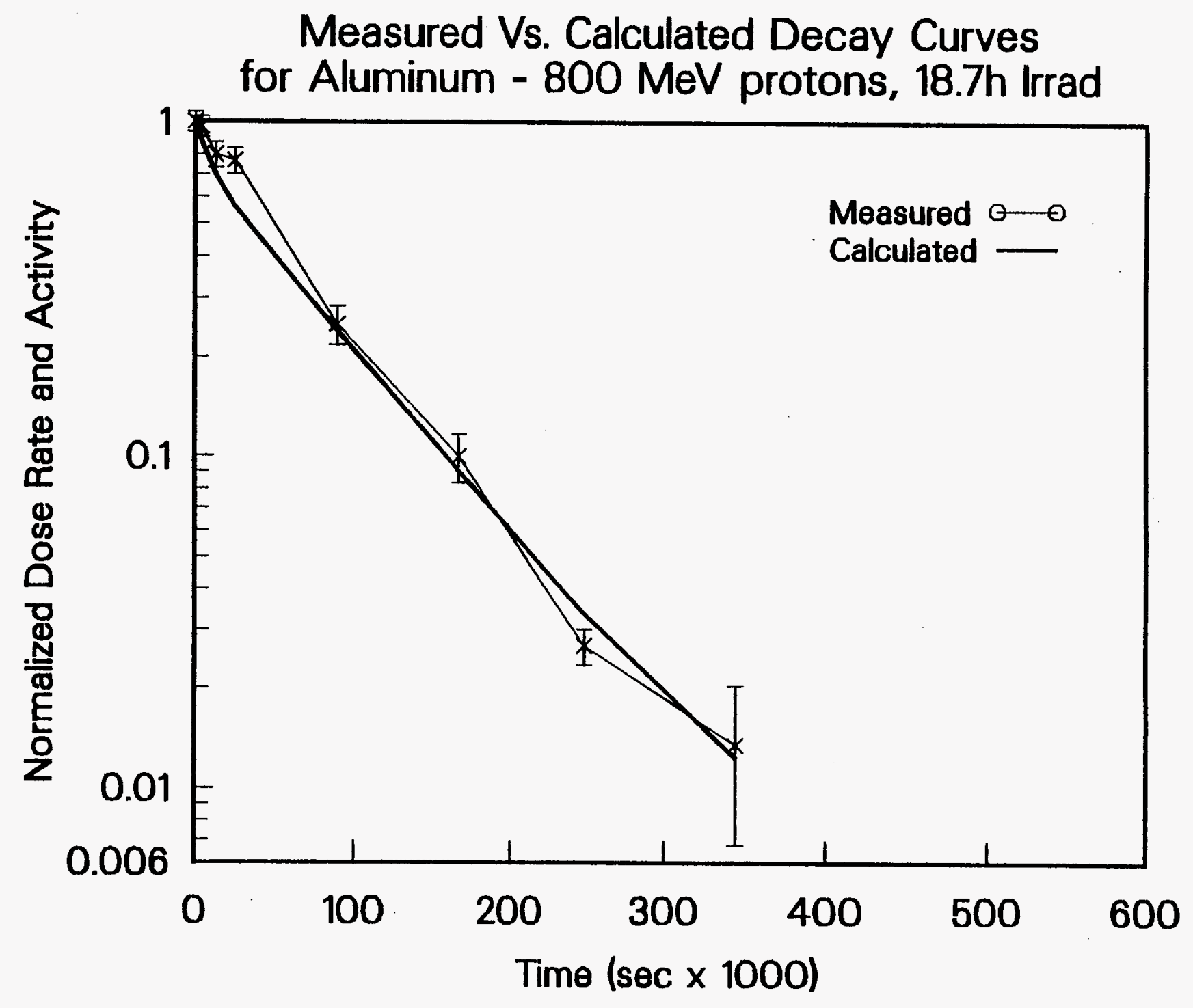

Figure 7 


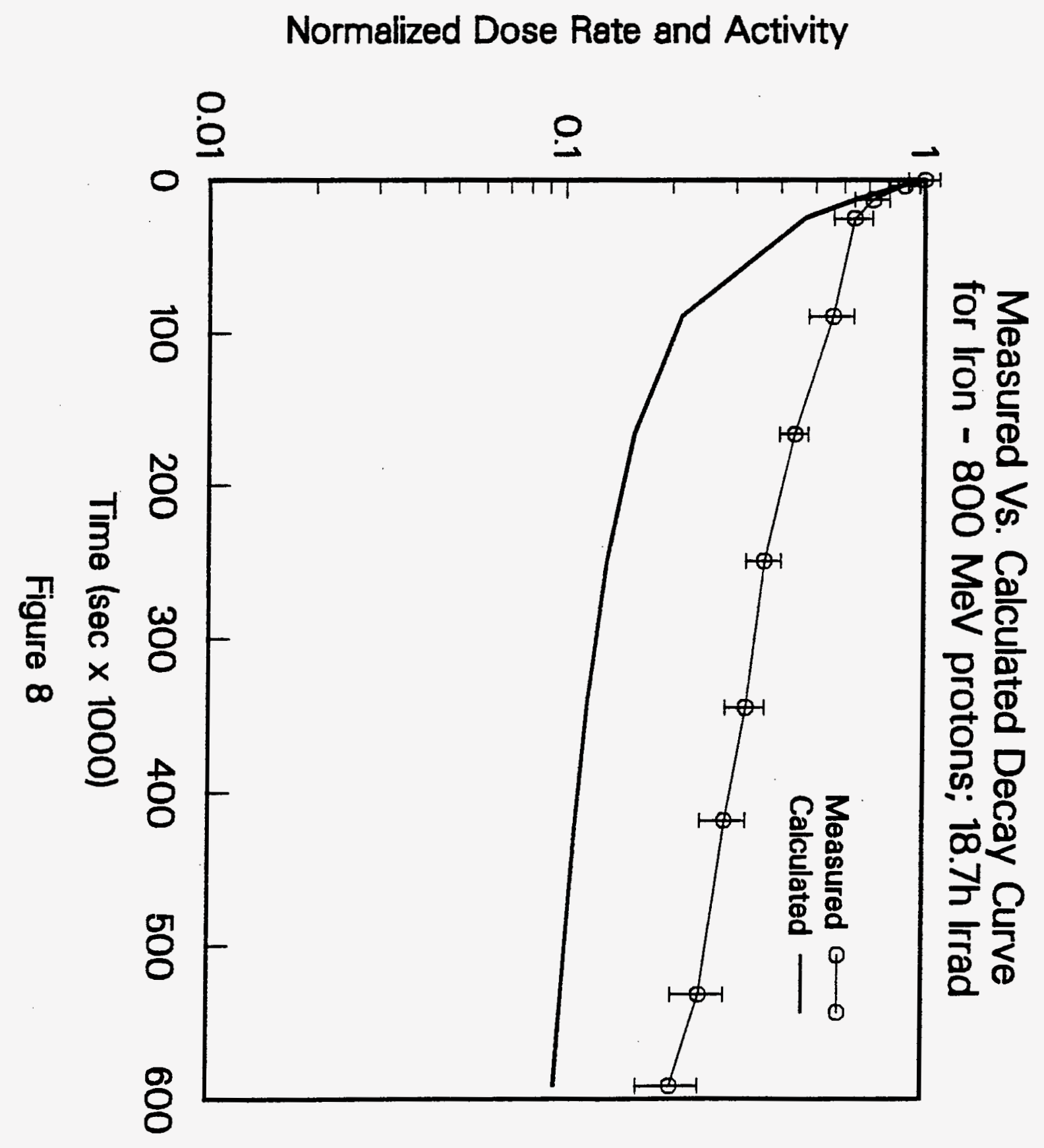




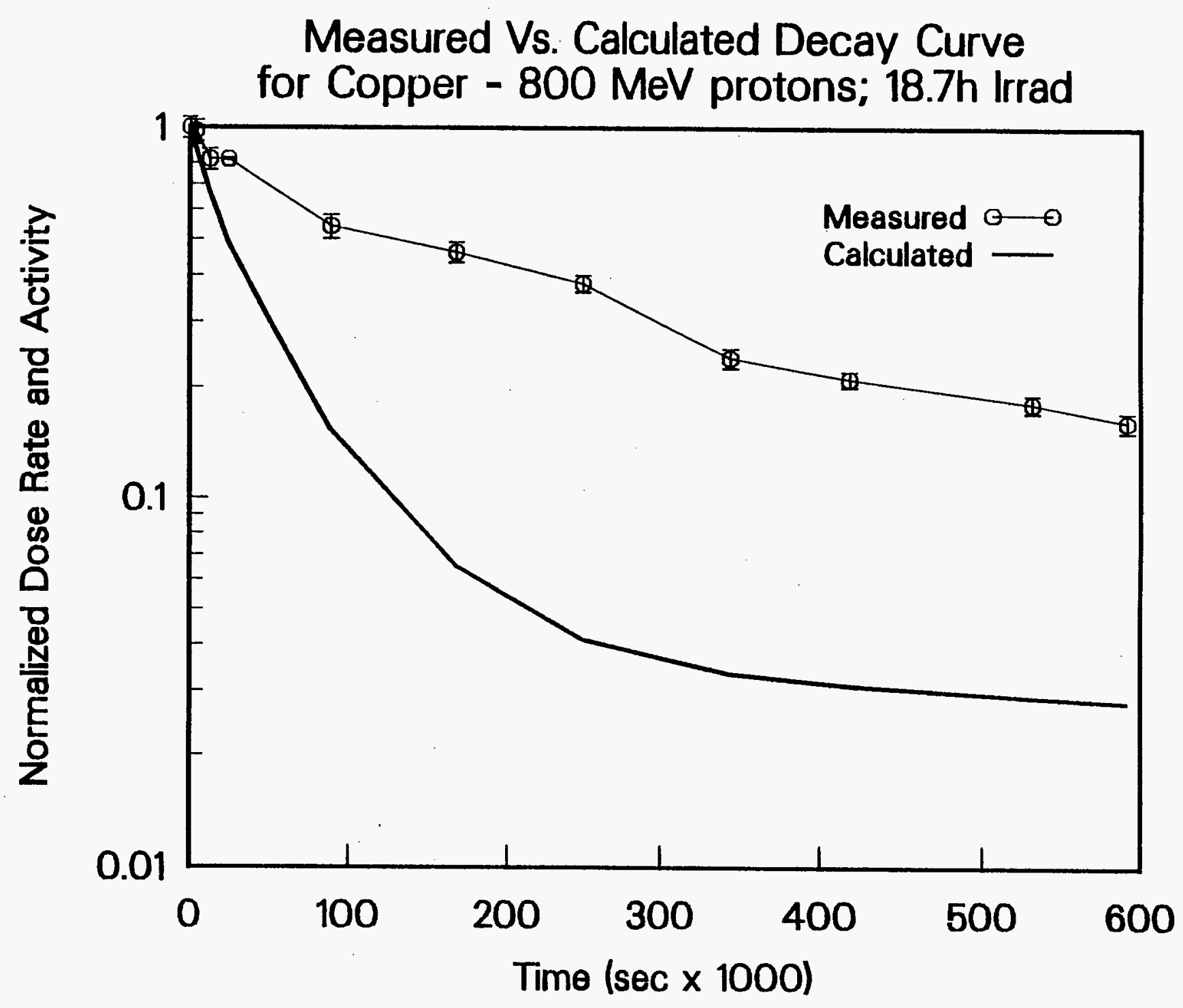

Figure 9 


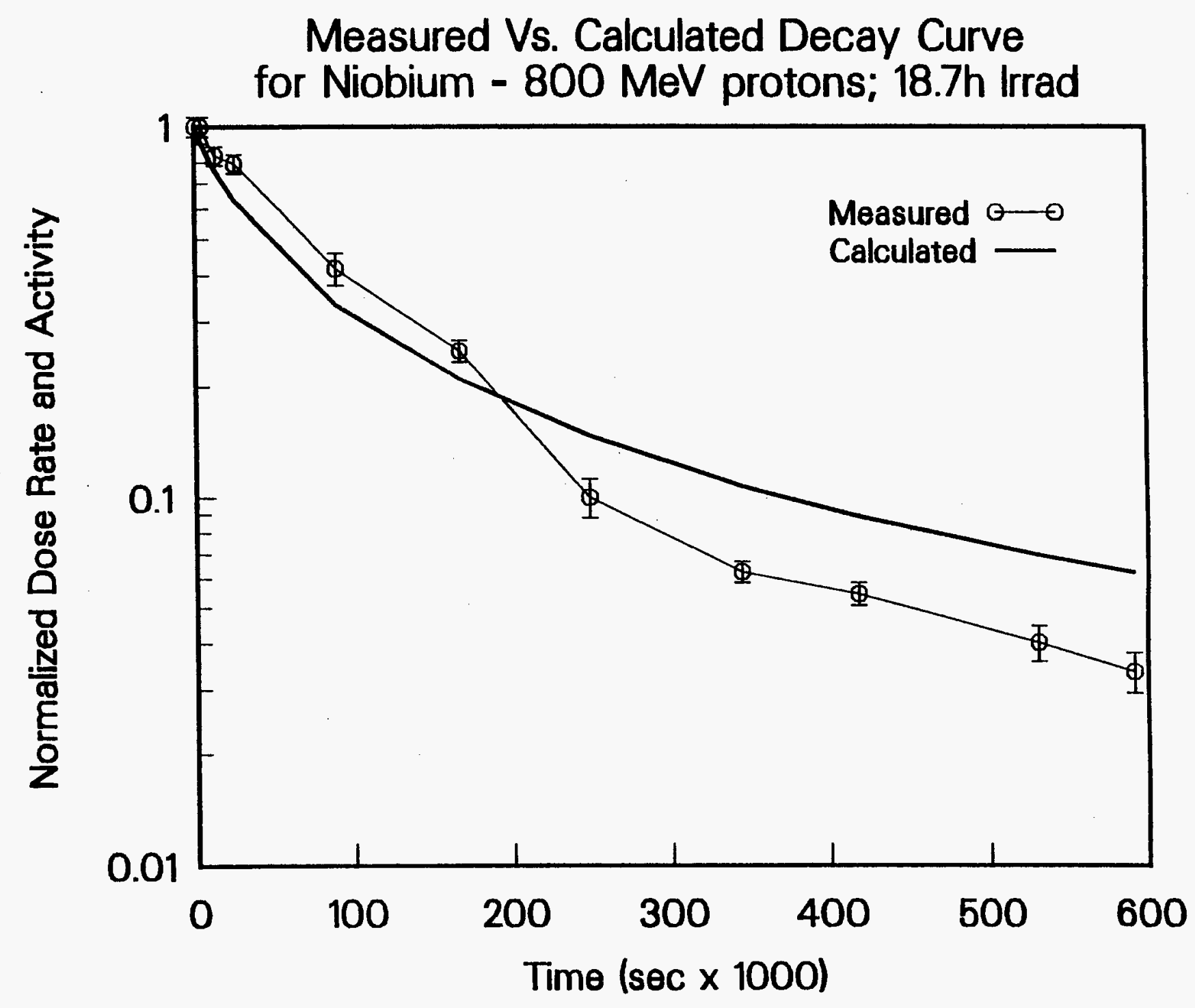

Figure 10 


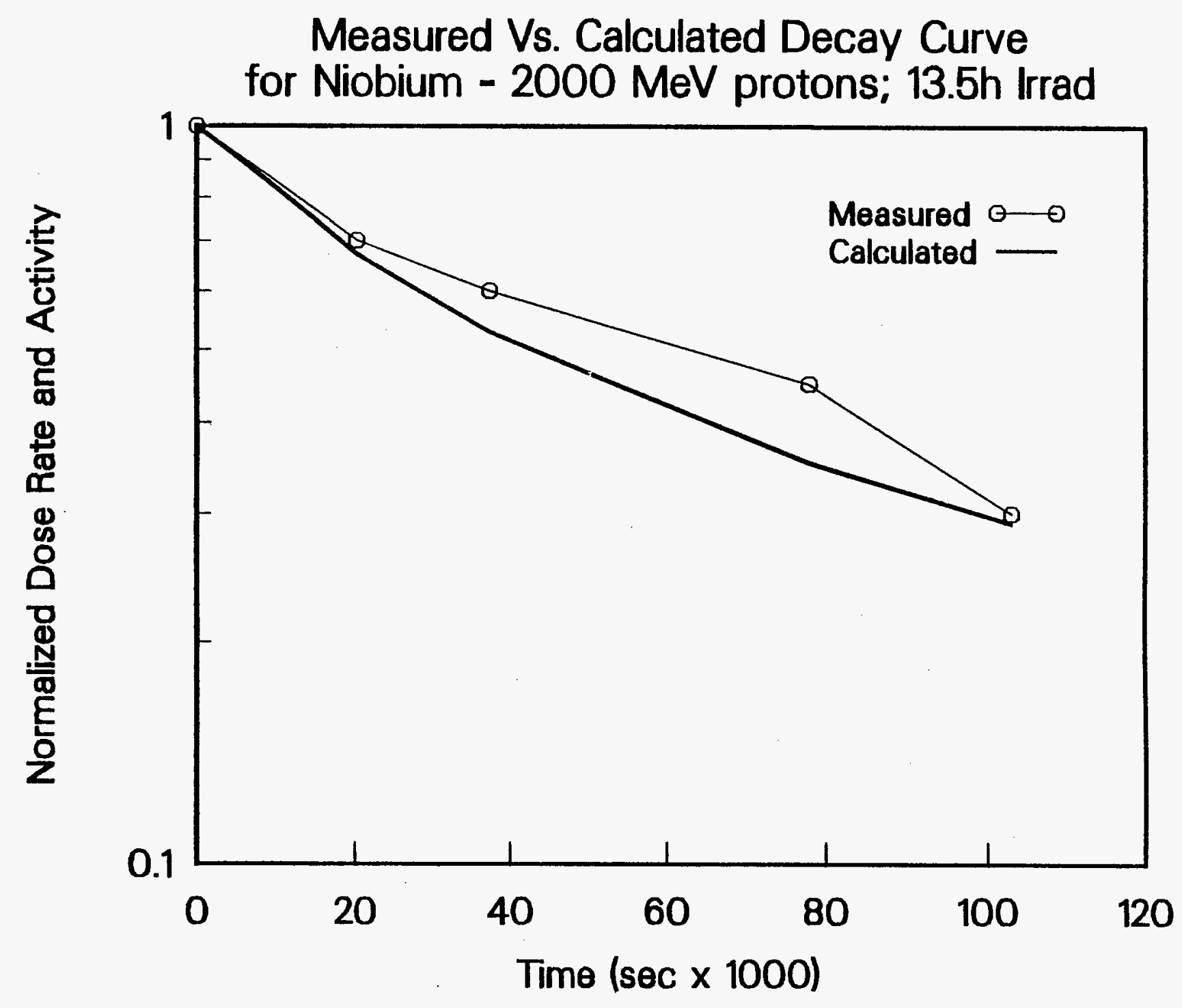

Figure 11 


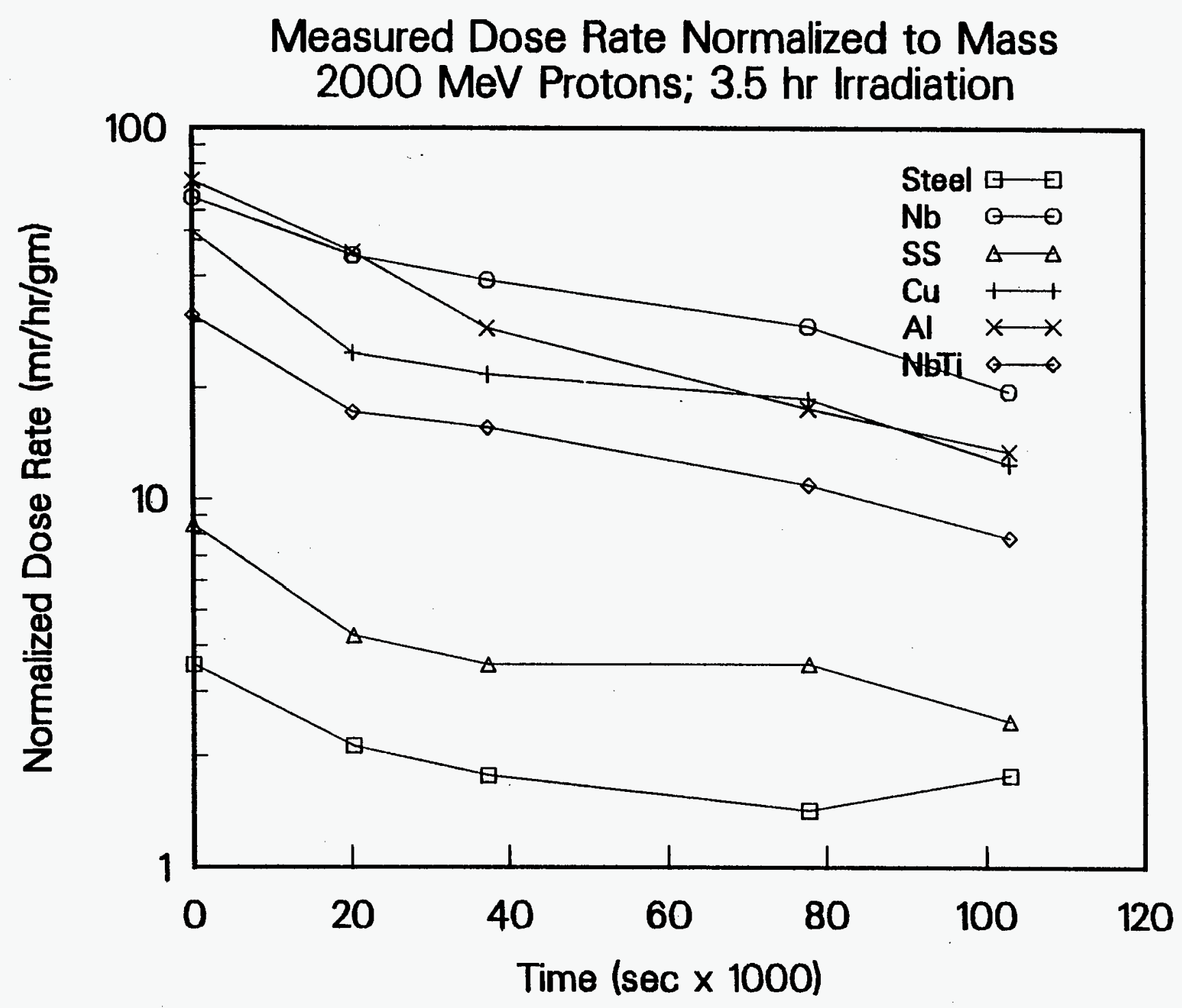

Figure 12 
Effective Half-life as a Function of Irradiation Time for $800 \mathrm{MeV}$ Protons

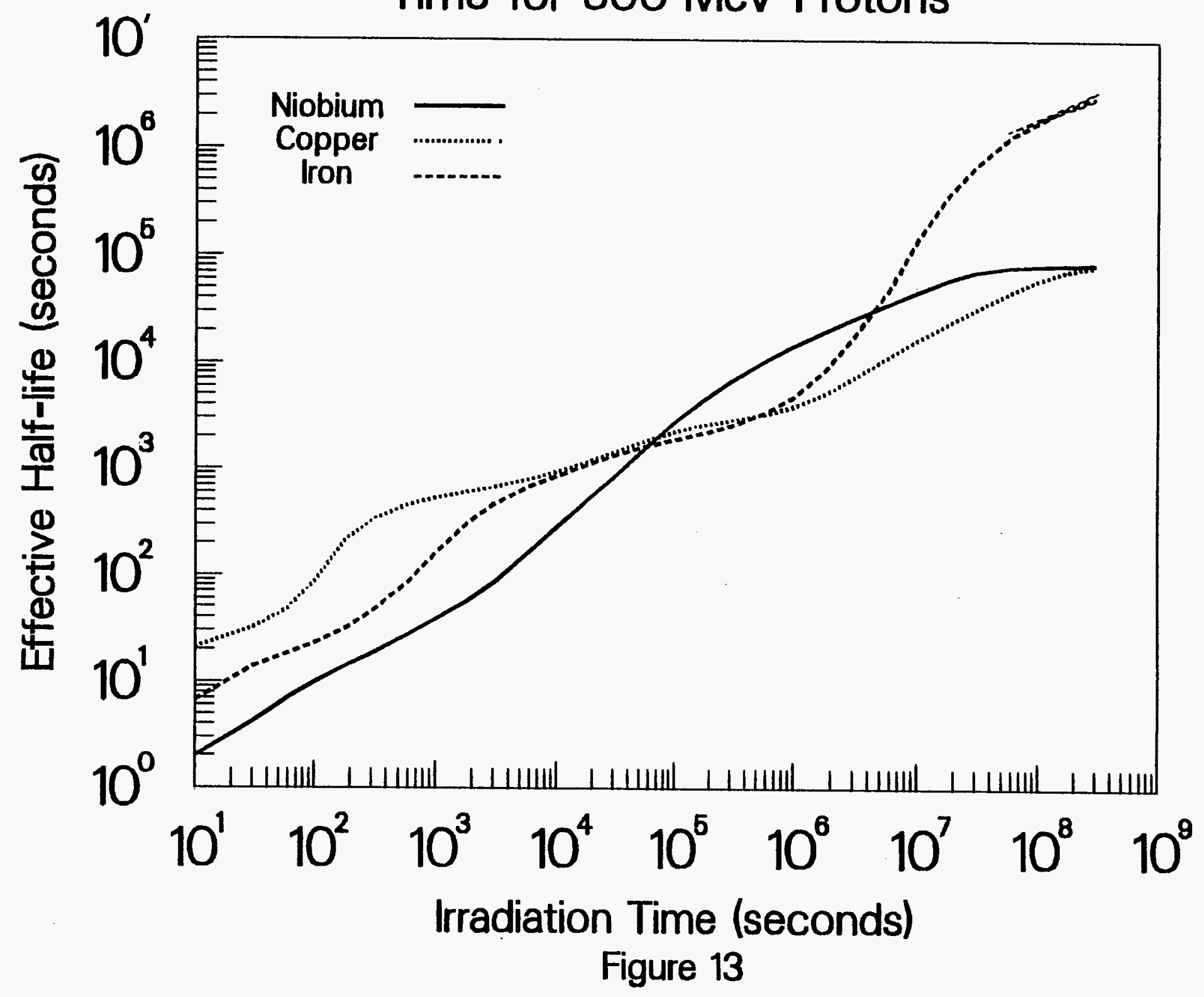




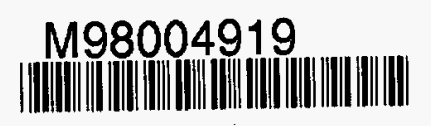

Report Number (14) BNL - 65396

Publ. Date (11) $\frac{199801}{D 0 E / D P, X F}$
Sponsor Code (18)
UC Category (19) UC.704, DOE/ER 